\title{
Editorial, special issue RE 2018
}

\author{
Daniel Amyot ${ }^{1} \cdot$ Walid Maalej $^{2}$
}

Published online: 10 July 2019

(c) Springer-Verlag London Ltd., part of Springer Nature 2019

The IEEE International Conference on Requirements Engineering (RE) is the premier conference on all aspects of requirements-related research. As in previous years, we continue a long-standing tradition and invited the authors of the best RE papers to submit an extended version to the Requirements Engineering Journal (REJ). This year, we have the pleasure to showcase four papers: three from the main RE research track and one from the industrial innovation track.

RE'18, the 26th installment of the RE conference, was held at the Banff Centre for Arts and Creativity in Canada's Banff National Park, known for its breathtaking mountainous surroundings, wildlife, and hot springs. The conference attracted over 250 researchers, practitioners, educators, and students to present and discuss the most recent research results, innovations, experiences, and concerns in the discipline of requirements engineering. On behalf of all RE'18 attendees, we thank and congratulate the entire organization team, led by the General Chair Guenther Ruhe, for hosting such an outstanding and memorable event. We also thank the reviewers, authors, organizers, volunteers, sponsors, and participants for their contributions and for their commitment to excellence in requirements engineering research and practice.

The RE' 18 main conference attracted 182 paper submissions, including 90 in the research track, 22 in the industrial innovation track, and 70 in the other tracks reporting on work in progress (RE@Next!, data, doctoral symposiums, posters/ demos, and panels). For the first time at RE, the research track used a double-blind reviewing approach where the authors' names were unknown to the reviewers. The Program Committee (PC) met in person in Hamburg, Germany, and discussed each paper with at least one positive review.

\footnotetext{
Daniel Amyot

damyot@uottawa.ca

Walid Maalej

maalej@informatik.uni-hamburg.de

University of Ottawa, Ottawa, Canada

2 University of Hamburg, Hamburg, Germany
}

In total, 24 research papers were accepted. The industry papers at RE' 18 were renamed Industry Innovation papers to stress the nature and importance of these contributions to the RE community. Each paper was also evaluated by three PC members, with an online discussion led by the Industry Innovation co-chairs: Lionel Briand and Smita Ghaisas. A total of eight papers were finally accepted. The four research papers with the best review scores and explicit support of the $\mathrm{PC}$, together with the best industry innovation paper, were invited to submit an extended version to this special issue of REJ. Four out of five groups of authors accepted the invitation. The extended papers went again through a rigorous review process, albeit single-blind given that the original papers were available and their authors known. Each extension had to be substantial, with over $40 \%$ of new material/results. Reviewers' comments on the original submissions also had to be taken into consideration. Each extended submission had two original and one new reviewer. After several iterations to meet the quality standards of the journal, all four papers were finally accepted.

In 2018, the RE conference theme was "Crossing Boundaries and Increasing Impact." While being focused on requirements engineering, we also aimed to open the door to innovation and new perspectives on the discipline. We believe that RE is inherently multidisciplinary: interfacing problems understanding and solutions design while involving various stakeholders. Yet, the boundaries between academia and industry, as well as those between software engineering and other areas, still deserve more attention. Crossing such boundaries and others between different methods and different application domains, such as automotive or e-commerce, can help increase the impact of RE research and techniques on practitioners' lives and on society in general. Not only was the theme well reflected in the conference program (e.g., in the excellent keynote presentations of Jane Cleland-Huang, Gail C. Murphy, and Krzysztof Czarnecki), but it is also reflected in the four papers of this special issue.

The first paper is "Teaching Requirements Elicitation Interviews: an Empirical Study of Learning from Mistakes," by Muneera Bano, Didar Zowghi, Alessio Ferrari, Paola 
Spoletini, and Beatrice Donati. This paper reports on a study of common mistakes in requirement elicitation interviews. The authors conducted an experiment with first-year students of a Master's program, focusing on identifying typical errors of novice interviewers with the goal of improving RE education.

In the second paper, "Identifying Incompleteness in Privacy Policy Goals using Semantic Frames," Jaspreet Bhatia, Morgan C. Evans, and Travis D. Breaux analyze privacy policies with the help of a technique called "semantic frames" in order to detect incomplete privacy statements, which can undermine user comprehension and affect the user's perceived privacy risks. The authors report on a grounded analysis done to identify the semantic roles in single policy statements, possible values for the semantic roles, their lexical and syntactic triggers, and how users may be willing to accept a policy depending on the presence of semantic roles.

The third paper, authored by S. Magnus Ågren, Eric Knauss, Rogardt Heldal, Patrizio Pelliccione, Gösta Malmqvist, and Jonas Bodénon, focuses on the "Impact of Requirements on Automotive Systems Development Speed." This paper addresses the managers' perspective on RE practices and development speed in the automotive domain. The authors have conducted a large qualitative study investigating what changes managers think are necessary to improve development speed. The paper also reflects on which of these challenges are addressed by the transition to agile development practices.
Finally, the best paper from the industrial innovation is "Automating Requirements Analysis and Test Case Generation," by Abha Moitra, Kit Siu, Andrew W. Crapo, Michael Durling, Meng Li, Panagiotis Manolios, Michael Meiners, and Craig McMillan. This paper introduces ASSERT, a requirements correctness and verification tool that has been successfully deployed in a large corporation. The paper introduces the tool features (e.g., to catch inconsistencies such as "wait $10 \mathrm{~s}$ " and "wait $100 \mathrm{~s}$ ") and illustrates through examples how to write an ontology and theorem and how to translate the documents into the ACL2 theorem prover for correctness analysis. The paper then explains how useful test cases can be generated.

We hope you will enjoy this special issue. We thank the authors and reviewers for their professional work and contributions. We are also thankful to Pericles Loucopoulos (REJ Editor in Chief), Valli Paramasivan from Springer Nature, and their production team for their help and support in turning this special issue into reality.

Publisher's Note Springer Nature remains neutral with regard to jurisdictional claims in published maps and institutional affiliations. 